[pp.85-91]

Vol. 4(1), Enero - Junio, 2016

http://dx.doi.org/10.15359/rnh.4-1.6

\title{
Actividad física. ejercicio físico y adulto mayor
}

Physical Activities. Physical Exercise and Older Adult

Ma Antonieta Ozols R

Universidad Nacional,

Costa Rica

antonieta.ozols.rosales@una.cr

Ma Antonieta Corrales A.

Universidad Nacional,

Costa Rica

antonieta.corrales.araya@una.cr

\section{Resumen}

Se ha comprobado que la actividad física y el ejercicio mejoran la calidad de vida de las personas mayores. Las investigaciones han encontrado progresos en el plano físico, emocional y social. Una de las organizaciones que está promoviendo estilos de vida saludables es la Organización Mundial de la Salud, que ha enfocado su atención en todas las etapas de la vida: desde la niñez hasta la edad adulta mayor.

Palabras clave: persona adulta mayor, ejercicio físico, actividad física.

\section{Abstracts}

It is proved that exercise and physical activity improve older people's quality of life. Investigations have found improvements in physical, emotional and social domains. One of the organizations promoting healthy life styles is the WHO, focusing its attention on all life stages, from childhood to old age.

Keywords: Older adult, physical exercise, physical activity.

A nivel mundial, el envejecimiento de la población es un fenómeno que ha sufrido cambios sustanciales en lo económico, social y cultural (Aranibar, 2001). El aumento en la expectativa de vida, descensos en la mortalidad infantil y 
disminución en la fecundidad son factores que han provocado el aumento de la población mayor en el mundo. Se espera que en 50 años, la población mayor sea de 58 millones, lo que equivale aproximadamente un $23,6 \%$ del total de la población (Kinsella, 2008).

Se entiende el envejecimiento como un "fenómeno altamente complejo y variable de una determinada especie, progresivo, común a todos los miembros, que involucra mecanismos de deterioro que afectan la capacidad de desempeñar un número de funciones" (Heikkinen, citado en Mahecha, 2001, p. 18).

Otra definición de envejecimiento indica que es un proceso fisiológico que no necesariamente va acorde con la edad cronológica y presenta variabilidad individual (Kuroda \& Israel, 1988), que abarca toda la vida, desde que se nace hasta la muerte.

El envejecimiento va a presentar deterioro en diferentes capacidades que determinarán la funcionalidad del individuo y, por ende, su calidad de vida. Se presenta disminución en la fuerza muscular, disminución en la densidad ósea, la capacidad de regeneración del músculo, entre otros. La fuerza es importante porque es utilizada para realizar diferentes acciones de la vida diaria, como: subir escaleras, levantarse de una silla o pasear.

La actividad física va a ser definida como cualquier movimiento de los músculos esqueléticos en los que se produce un gasto energético. Por su parte, el ejercicio físico será entendido como la realización de movimientos corporales planificados, repetitivos y supervisados.

La actividad física ha demostrado mejorar sustancialmente la capacidad funcional de la persona mayor, se encuentra que las posibilidades de adquirir enfermedades crónicas o cardiovasculares disminuyen y se presenta una reducción de las medidas antropométricas (peso) y control de las enfermedades respiratorias.

El ejercicio beneficia a la persona adulta mayor que padece enfermedades crónicas respiratorias al disminuir su incidencia, al realizar ejercicios en los brazos y piernas, después de un programa de rehabilitación respiratorio.

En el ámbito psicológico se han encontrado efectos positivos en la salud mental (Mahecha, 2001) que provocan un aumento en el autoconcepto, disminución en 
la ingesta de medicamentos, mejoras en la tensión muscular, y el insomnio y una mejora en las funciones cognitivas y de socialización (Mahecha, 2001).

Una de las enfermedades mentales más común en el sujeto adulto mayor es la depresión, reportándose una disminución significativa después de realizar actividad física (Mahecha, 2001).

Mora, Villalobos, Araya y Ozols (2004) realizaron un estudio para conocer la relación entre las variables (soporte social, autonomía, salud mental, actividad físico recreativa) que integran la perspectiva subjetiva de la calidad de vida, ligadas al género en personas adultas mayores costarricenses. Aunado a esto el principal motivo para realizar la actividad física y el beneficio percibido por estos, de acuerdo con el género. La muestra del estudio fue 152 sujetos ( 92 mujeres y 60 hombres con edades entre los 60 a 75 años, de 21 centros diurnos a los cuales se les aplicó dos instrumentos: un cuestionario de calidad de vida (INISA, 1994) y una encuesta sobre actividad física recreativa. Según los resultados encontrados se puede concluir que la calidad de vida (autonomía, soporte social, salud mental, actividad física recreativa) difiere en lo que respecta al género. La depresión geriátrica presentó diferencias significativas en cuanto al género que es superior en las mujeres. En cuanto al motivo para realizar actividad física recreativa ambos coincidieron que era por salud, ya que se sentían reanimados, aliviados, más alegres y con energía. Las personas adultas mayores que practicaban diariamente actividad física presentaron un mejor nivel de calidad de vida en relación con los sujetos sedentarios.

Bludau (2010) reportó varios beneficios de la actividad física y el ejercicio sobre la persona mayor: el caminar mejora la forma de caminar y evita accidentes con caídas. El ejercicio es uno de los principales tratamientos para problemas vasculares periféricos porque aumenta la cantidad de sangre que es movilizada disminuyendo el dolor causado por la caminata.

El ejercicio puede disminuir los niveles de azúcar en la sangre en las personas diabéticas, reducción del peso, el colesterol y control de la presión arterial. En personas con osteoporosis se ha encontrado un aumento en la densidad del hueso (Bludau, 2010). Otros estudios han encontrado que el ejercicio físico ayuda a conservar la masa ósea, evitando las fracturas que limitan su independencia (López, 2014).

La evidencia científica ha encontrado que la actividad física puede disminuir la aparición del cáncer. Fruth (2003) investigó sobre el efecto del ejercicio para 
determinar si mayores niveles de actividad física presentaban menor incidencia de cáncer comparadas con aquellas con menores niveles de actividad. Al finalizar su estudio encontró que no existía una asociación entre el nivel de actividad física de las personas, su grado de actividad física y la incidencia de cáncer. Por otro lado, otros estudios han encontrado una asociación positiva en algunos tipos de cáncer: colon, mama, pulmón, páncreas y próstata. Se concluye que realizar actividad física en la edad adulta reduce el riesgo de cáncer en comparación con las personas sedentarias.

Salinas y otros (2005) realizaron un estudio observacional de 821 personas adultas y adultas mayores en control en 40 consultorios de atención primaria incorporadas al programa piloto de actividad física integral, pertenecientes a las 13 regiones del país. Los sujetos ingresaron al programa en control de salud cardiovascular-adultos de 18 a 59 años y adultos mayores independientes de 60 o más años portadores de uno a más factores de riesgo cardiovascular (hipertensión arterial, diabetes tipo II, obesidad), sin contraindicaciones para el ejercicio y que aceptaron participar libre e informadamente. El período de conformación de los grupos varió dependiendo de la región, duró aproximadamente tres meses en todo el país.

El programa piloto estuvo conformado principalmente de talleres de actividad física grupal (20 participantes) de ocho meses de duración, con una frecuencia de tres veces a la semana, cada sesión de 60 minutos (total de 96 sesiones). Además de la actividad física se desarrollaron charlas informativas sobre estilos de vida saludable que complementaron las sesiones. La metodología incluyó los conceptos de aprender haciendo y aprender reflexivo, fomentó la responsabilidad y el apoyo colectivo y fue diseñada considerando diversos modelos teóricos de cambio conductual, de educación participativa y planificación comunitaria. Los resultados mostraron que un $98,4 \%$ de los usuarios y usuarias respondió sentirse mejor después de haber participado, un $98,8 \%$ consideró muy adecuado el esfuerzo físico realizado y un $88,5 \%$ estuvo muy satisfecho con las actividades realizadas.

Al preguntárseles si mantendrían las conductas de vida activa adquiridas, se observó que un $73 \%$ de participantes manifestó la intención de continuar caminando 30 minutos diarios, un $60 \%$ realizar ejercicio tres veces por semana, 30 minutos cada vez y un $38 \%$ recrearse activamente. Concluyendo, se encontró que este tipo de programas son efectivos para desarrollar estilos de vida saludable en las personas adultas mayores. 
Se han encontrado beneficios no solo en la persona mayor que no tiene una discapacidad, sino también en las personas que adquieren una discapacidad.

Pérez, Fernández, Martínez, Ochoa y Velázquez (2004) aplicaron un programa de ejercicios que involucró equilibrio, coordinación y acondicionamiento durante seis meses en personas con problemas neurológicos, específicamente ataxia espinocerebelosa tipo 2 (SCA2). Estos investigadores encontraron resultados significativos en la mejora del equilibrio y en los indicadores neurológicos con los ojos abiertos y ojos cerrados.

Otro estudio similar fue realizado en personas con Parkinson, en este aplicaron un programa de ejercicio acuático para determinar el efecto sobre la cinemática del patrón de marcha. Los resultados fueron significativos en la velocidad de marcha, longitud de la zancada, tiempo del paso y en la relación apoyo simple, apoyo doble. Estos datos nos indicaron que el ejercicio acuático produce efectos positivos sobre las variables cinemáticas que caracterizan el patrón de biomecánica de pacientes con problemas neurológicos como Parkinson (Rodríguez, Cancela, Ayán, Nasocimineto, Seijo-Martinez, 2013).

Barrios, Borges y Cardoso (2003) evaluaron los beneficios percibidos de la persona adulta mayor sobre el ejercicio y se encontró que el ejercicio contribuye a: disminuir los dolores, calambres y entumecimientos, mejora el sueño, fortalecimiento de las piernas y el cuerpo en general, mejora el estado de ánimo y distracción. Y para concluir encontraron que las contribuciones físicas y sociológicas las expresaron en relación con sus vivencias.

Campos et al. (2003) realizaron un estudio de los efectos de la aplicación de un programa de ejercicio físico basado en el desarrollo de la fuerza sobre tres variables del bienestar psicológico: el autoconcepto físico, la autoestima global y la satisfacción con la vida. Los sujetos del estudio fueron 90 mujeres mayores de 55 años que formaban parte de los programas de actividad física en diferentes municipios de la Comunidad Valenciana. El programa de ejercicio consistió en un entrenamiento por circuito de fuerza, durante un período de seis meses. Al finalizar el programa produjo un incremento significativo en los niveles de fuerza y en todas las variables psicológicas.

En conclusión, la actividad física y el ejercicio contribuyen significativamente en la calidad de vida de la persona adulta mayor proveyendo mejores condiciones físicas, sociales y emocionales para enfrentar el proceso de envejecimiento. 


\section{Referencias}

Aranibar, P. (2001). Acercamiento conceptual a la situación del adulto mayor en América Latina. Proyecto Regional de Población CELADE-FNUP- Centro Latinoamericano y Caribeño de Demografía (CELADE). Santiago, Chile.

Barrios, R., Borges, R. y Cardoso, L. (2003). Beneficios percibidos por adultos mayores incorporados al ejercicio. Revista Cubana de Medicina General Integral, 19(2).

Bludau, J. (2010). Aging but never old: the Realities, myths, and misrepresentations of the aging movement. Santa Bárbara, California: Praeger.

Campos, J., Huertas, F., Colado, J. C., López, A. L., Pablos, A \& Pablos, C. (2003). Efectos de un programa de ejercicio físico sobre el bienestar psicológico de mujeres mayores de 55 años. Revista de Psicología del Deporte, 12(1), 7-26.

Fruth, L. (2003). Physicl activity and incidence of cancer: A metanalysis: Unpublished Doctoral (PhD). The University of Toledo, USA.

INISA. (1994). Validación de un instrumento para la medición de la calidad de vida de personas de 60 años y más. Programa de Investigación sobre el envejecimiento. San José, Costa Rica: Editorial UCR.

Kinsella, K y Wan, H. (2009). An aging world: 2008. International Population Reports. Washington, U.S: Census Bureau. Recuperado de http://www. census.gov/prod/2009pubs/p95-09-1.pdf

Kuroda, Y. \& Israell, S. (1988). Sport and physical activities in older people. In A. Dirix, et al. (Eds). The Olympic book of sports medicine (pp. 331-355, 1st ed.). Oxford: Blackwell Scientific Publications.

López, J. (2014). Actividad fisica aplicada a la osteoporosis. España, Barcelona: Elsevier.

Mahecha, S. A. (2001). Envelhecimento \& Atividade fisica. Centro de Estudos do Laboratorio de aptidao física de Sao Caetano du Sul. Brasil

Mora, M., Villalobos, D., Araya, G. y Ozols, M. A. (2004). Perspectiva subjetiva de la calidad de vida del adulto mayor, diferencias ligadas al género y a la 
práctica de la actividad físico recreativa. Revista MHSalud, 1(1),1-12.

Pérez, I., Fernández, j. A., Martínez, e., Ochoa, R. y Velázque, M. J. (2004). Efectos de un programa de ejercicios físicos sobre variables neurológicas cuantitativas en pacientes con ataxia espinocerebelosa tipo 2 en estadio leve. Revista de Neurología, 39(10), 907-10.

Salinas, J. Bello, M, Flores, A., Carbullanca, L \& Torres, M. (2005). Actividad fisica integral con adultos y adultos mayores en Chile: Resultados de un programa piloto. Rev. Chil. Nutr. 32(3). doi: http://dx.doi.org/10.4067/S071775182005000300006 .

Rodríguez, P., Cancela, J. M., Ayán, C., Do Nascimento, C. y Seijo-Martinez, M. (2013). Efecto del ejercicio acuático sobre la cinemática del patrón de marcha en pacientes con enfermedad de Parkinson. Un estudio piloto. Revista Neurología, 56(6), 315-320 